\title{
Proceedings of the 138th Semon Club, 20 November 2009, Otolaryngology Department, Guy's and St Thomas' NHS Foundation Trust, London, UK
}

\author{
Chairman: Miss Elfy B Chevretton, Guy's and St Thomas' NHS Foundation Trust. \\ Secretary: Mr Sherif Haikel, Guy's and St Thomas' NHS Foundation Trust. \\ Pathologists: Professor Leslie Michaels and Dr Ann Sandison, University College London. \\ Radiologists: Dr Steve Connor and Dr Ata Siddiqui, Guy's and St Thomas' NHS Foundation Trust. \\ Professor Leslie Michaels awarded a prize for the best presentation of the meeting to Mr R A Tasca.
}

\section{Head and neck session}

Chairman: Mr J P Jeannon

\author{
A large skull base tumour with acute urinary retention \\ A Mohan, A Balfour, R Simo \\ From Guy's and St Thomas' Hospital NHS Trust, London
}

\section{Background}

A previously healthy, 49-year-old man presented to the accident and emergency department with a short history of severe headaches, neck pain and intermittent blurred vision. Initial computed tomography scanning was reported as showing a suspected nasopharyngeal carcinoma, and the patient was referred to the ENT out-patient clinic. He had no symptoms of nasal obstruction or cervical lymphadenopathy, and no evidence of disease in the postnasal space on examination. A repeated scan demonstrated a large, destructive skull base tumour involving the body of the sphenoid, clivus, petrous apices and cavernous portion of the carotid canal. Within one week of admission, the patient's symptoms progressed rapidly.

\section{Radiology}

Computed tomography, magnetic resonance imaging and bone scans demonstrated rapid local progression of disease, with soft tissue invasion and expansive bony and lytic lesions of the skull vault, mandible and seventh thoracic vertebra.

\section{Histology}

Biopsies from the seventh thoracic vertebra confirmed lymphomatous adult T-cell lymphoma/leukaemia.

\section{Management}

The patient was initially admitted for pain control and diagnostic investigation. The head and neck multi-disciplinary team initially planned to perform a tissue biopsy via the trans-sphenoidal approach, but the patient rapidly developed acute spinal cord compression with acute urinary retention. This required thoracic spine stabilisation by the orthopaedic team; biopsies were taken simultaneously. Following diagnosis, the patient was transferred to the haematology team and commenced on chemotherapy with cyclophosphamide, doxorubicin (Adriamycin), vincristine (Oncovin) and prednisolone, together with anti-retroviral treatment.

\section{Conclusion}

Adult T-cell lymphoma/leukaemia has been described extensively in the literature. However, isolated bony involvement without systemic signs or lymphadenopathy is a rare presentation. The presented case was very aggressive in nature, and highlights the diagnostic and practical challenges faced by the ENT surgeon; it also demonstrates a successful multi-disciplinary approach to management.

An uncommon midline neck lump

T G Ong, A K Golagani, A M Lale

From Dorset County Hospital NHS Foundation Trust, Dorchester

\section{Introduction}

Castleman's disease is a rare lymphoproliferative disorder which commonly affects the mediastinum but also occurs rarely in the head and neck.

\section{Case report}

A 39-year-old man presented with a three-year history of an asymptomatic submental neck lump which measured $8 \times$ $15 \mathrm{~mm}$ in size. The lump was surgically excised and the specimen sent for histopathological analysis.

\section{Radiological findings}

An ultrasound scan revealed appearances consistent with a submental lymph node, but without the fatty, echogenic hilum of a normal lymph node, suggesting that the lesion was pathological. A full body computed tomography scan found no other significant lymphadenopathy.

\section{Histological findings}

Histopathological examination revealed numerous large follicles with prominent proliferation and hyalinisation of the germinal centre. The interfollicular areas were composed of hyperplasic vessels and plasmacytoid dendritic $T$ cells. The findings indicated the hyaline vascular type of Castleman's disease.

\section{Conclusion}

Castleman's disease is a rare condition which should be considered in the differential diagnosis of a midline neck lump. A biopsy is required, as aspiration cytology is unlikely to provide the diagnosis. 
'I have a lump in my throat doctor'

M Whittaker, J R Tysome, J P Jeannon

From Guy's and St Thomas' Hospital NHS Trust, London

Background

We present the third reported case of an unusual mesenchymal tumour of the larynx.

\section{Case report}

A 74-year-old man presented with the sensation of a lump in his throat, together with intermittent aphonia. He denied any dysphagia or weight loss, but was a former heavy smoker. Flexible nasendoscopy revealed a long, smooth polyp arising from the left arytenoid.

\section{Radiological findings}

There was enlargement of the left pyriform sinus and aryepiglottic fold, with no enlargement of the cervical lymph nodes.

\section{Management}

The patient underwent microlaryngoscopy, during which a $7 \mathrm{~cm}$ polyp was excised from the lateral aspect of the left arytenoid.

\section{Histology}

On histopathological examination, the polyp showed a vascular, fibromyxoid stroma with chronic inflammatory infiltrate and lobules of adipose tissue. It was covered by stratified squamous epithelium. Professor Michaels commented that such non-specific inflammatory lesions can occur spontaneously, and labelled the presented polyp as a benign superficial angiomyxoma.

\section{Discussion}

Angiomyxoma is an uncommon mesenchymal tumour, most often found in the pelvis, with a six:one female to male ratio. To date, only a few cases have been reported in the larynx. Despite being described as a benign tumour, it may be locally aggressive with the potential to infiltrate muscle and fat. The prognosis is very good, but local recurrence is common and may occur several years after identification of the primary tumour.

\section{Subglottic lesion in a patient with a thyroid mass}

J Nichani, L Menasce, S Loughran

From The Central Manchester Foundation Trust and Christie Hospital NHS Trust, Manchester

\section{Introduction}

We present a case report of a patient with a thyroid mass and an incidental subglottic lesion.

\section{Case report}

A 44-year-old woman presented with a right thyroid mass. Ultrasound examination diagnosed this as a complex cyst. Fine needle aspiration cytology (FNAC) was carried out, and reported as showing a colloid cyst. Nasendoscopy also identified a subglottic mass. Computed tomography (CT) scanning of the neck was conducted. At microlaryngoscopy, a purple mass in the subglottis was biopsied; this was reported to comprise thyroid follicular cells with no evidence of malignancy. Repeated FNAC was reported as suspicious of malignancy (Thy3). Positron emission tomography scanning did not identify increased uptake in the thyroid gland or the trachea.

\section{Radiological findings}

A contrast-enhanced CT scan of the neck showed a large, heterogeneous thyroid mass. However, there was evidence of tracheal invasion from the right lobe of the thyroid, filling the right half of the subglottis and upper trachea.

\section{Histological findings}

The excised thyroid gland had the appearance of a nodular colloid goitre with hyperplasia. There was no evidence of necrosis or vascular invasion. The nodular changes seen in the thyroid tissue extended beyond the confines of the thyroid gland into the trachea and larynx, and possibly involved ectopic intra-tracheal thyroid tissue.

\section{Management}

Following discussion at the thyroid multi-disciplinary team meeting, this patient underwent total thyroidectomy, tracheal resection and reconstruction.

\section{Conclusion}

Evidence of intra-laryngotracheal thyroid tissue is most likely to be a result of invasive thyroid cancer. Intra-tracheal thyroid tissue as seen in this patient is a rare condition, and can only be diagnosed when histological findings are benign.

\section{Haemorrhagic swelling of the tongue}

R Madada-Nyakauru, S Watts

From Worthing Hospital, West Sussex

\section{Introduction}

Massive swelling of the tongue is potentially fatal. Haemorrhagic swelling is rare but can arise due to trauma, tumour, coagulopathies or arterio-venous malformations.

\section{Case report}

An 83-year-old, edentulous woman presented to the accident and emergency department with massive haemorrhagic engorgement of the tongue and sublingual area, which had been preceded by 4 hours of throat soreness for which she had taken paracetamol. On admission she rapidly became stridulous.

Past medical history included atrial fibrillation (treated with aspirin $300 \mathrm{mg}$ ) and thyroidectomy.

On assessment, the patient was hypertensive but other observations were normal. Examination showed gross, generalised submandibular swelling, with left-sided tenderness and no lymphadenopathy.

Nasendoscopy revealed haemorrhagic lesions and bullae along the lateral walls of the pharynx, with valecullar swelling. The vocal folds appeared normal.

The patient's haemoglobin level was $9.3 \mathrm{~g} / \mathrm{dl}$ and her salicylate concentration was $<0.1 \mathrm{mg} / 1$. Clotting parameters, platelet count and function, and $\mathrm{C} 1$ esterase inhibitor levels were normal, and plasmapheresis was negative.

\section{Radiological findings}

Computed tomography showed a prominent right submandibular gland and gross submandibular swelling. Neither lingual artery showed any specific abnormality or haemorrhage. No mass lesions were identified.

\section{Management}

Emergency surgical tracheostomy was performed. Further engorgement caused vascular compromise of the tongue, so a floor of mouth incision was made to allow haematoma 
evacuation and partial tongue decompression. The patient was managed in the intensive therapy unit.

\section{Conclusion}

Haemorrhage further complicates the management of tongue swelling. Protecting the airway is the priority. In our patient, anticoagulation with aspirin was the likely cause of a spontaneous arterial bleed. She made a full recovery and was discharged after three weeks.

\section{Otology and skull base session}

Chairman: Mr Tony Aymat

\section{A cholesteatoma... or not?}

B F Jones, J Deans, G Bockeler

From Leighton Hospital, Crewe

\section{Introduction}

Neuroglial heterotopia typically occurs within midline structures, classically the postnasal space, oropharynx, tongue and palate. Involvement of the middle ear is rare, and reported cases are associated with previous surgery or trauma, chronic infections, and inflammation.

\section{Case report}

A 55-year-old woman with unilateral otorrhoea and a conductive hearing loss was initially diagnosed with otitis externa. She was found to have an attic retraction pocket filled with keratin debris, and underwent a mastoid exploration. Intra-operative findings comprised granulation tissue within the attic. The tegmen was dehiscent, with what was thought to be further granulation tissue attached to the dura. A cerebrospinal fluid leak was recognised and repaired.

The tissue was found to be neural in origin, with a differential diagnosis of an encephalocoele or neuroglial heterotopia. Post-operative radiological imaging confirmed an extensive deficiency within the tegmen.

\section{Conclusion and panel discussion}

Histologically, there is no distinction between an encephalocoele and neuroglial heterotopia. The diagnosis is made based on the correlation between clinical findings and radiological imaging. All previously reported such patients who presented with an associated cholesteatoma had previously undergone surgery. The current case differs in that this was the patient's first presentation, with no history of surgery or trauma.

Professor Michaels agreed with the diagnosis of encephalocoele in this case, but noted that a choristoma of glial tissue is a more common cause of heterotopic tissue than heterotopia.

\section{An uncommon external auditory meatus mass presenting subacutely}

\section{N Grover, F Amine, G Brookes}

From the Royal National Throat, Nose and Ear Hospital, London

\section{Introduction}

Paraganglioma of the external auditory canal is a rare entity. We report a case in a 49-year-old woman, and discuss the potential pitfalls in diagnosis and treatment.
Case report

A 49-year-old African woman presented with a three-week history of left-sided otalgia, hearing loss and tinnitus. Examination revealed a firm swelling arising from the superior portion of the left external auditory canal. Pure tone audiometry showed an ipsilateral conductive hearing loss of $25 \mathrm{~dB}$. Having made a provisional diagnosis of otitis externa, the patient was managed with antibiotic ear drops and a Pope wick. Mr Aymat noted that the lack of otorrhoea argued against our provisional diagnosis. Attempted aspiration of the swelling resulted in bleeding, which was controlled by packing.

\section{Radiological findings}

A computed tomography scan of the temporal bones revealed a non-specific soft tissue mass in the external auditory canal, with a thickened tympanic membrane and subtle erosion of the superior aspect of the bony canal.

\section{Histological findings}

A biopsy was performed under general anaesthetic. Histopathological examination showed a proliferation of cells which showed immunohistochemical staining properties consistent with a paraganglioma.

Professor Michaels and Dr Sandison agreed that, histologically, the lesion appeared to be a neuroendocrine tumour.

\section{Management}

Subsequently, the mass was completely excised via an endaural approach. Six months post-operatively, the patient remained clinically free of recurrence

\section{Post-traumatic sudden vertigo and left-sided} sensorineural hearing loss

A Espeso, M Prior

From Derriford Hospital, Plymouth

\section{Case presentation}

A 69-year-old woman presented with a sudden onset of vertigo and reduced hearing, following falling onto the shaft of a comb while scratching her left ear.

The patient was found to have a negative fistula test, a positive Romberg's test and no nystagmus. An urgent pure tone audiogram showed a left-sided, moderate to severe, sensorineural hearing loss. The patient was admitted for symptomatic relief, which was achieved after 48 hours. Nine days later, she was admitted again with recurrent vertigo and a deterioration in her hearing. A computed tomography (CT) scan of the temporal bones showed evidence of free air within the left labyrinth.

An emergency exploratory tympanotomy demonstrated an open vestibule due to dislocation of the stapes. The defect was packed with adipose tissue.

\section{Discussion}

Pneumolabyrinth is a condition in which air is present in the vestibule and/or the cochlea. Dr Connor demonstrated evidence of air in this patient's vestibule on the high resolution CT scan, predicting a perilymphatic fistula. Mr Aymat and Miss Chevretton discussed the urgency of the case and the need for immediate CT scanning in traumatic cases. In addition, Mr Aymat, Miss Chevretton and several attendees contributed to the discussion of different surgical approaches and techniques, complimenting the novel use of adipose tissue for repair. 
An unusual cause of chronic otorrhoea

N De Zoysa, J Stephens, P Kothari

From Luton and Dunstable Hospital, Bedfordshire

\section{Case presentation}

A 41-year-old woman with type I diabetes presented with a chronically discharging ear. Examination revealed the presence of granulations in the distal external auditory canal. Biopsies of the external auditory canal showed no evidence of malignancy. Initial computed tomography (CT) scanning showed only slight thickening of the tympanic membrane.

A cortical mastoidectomy and tympanoplasty were undertaken, during which the tympanic membrane was resected. Histological examination showed tissue invaded by squamous cell carcinoma.

Professor Michaels reviewed the slides and agreed with the diagnosis. He further commented that this finding was highly unusual without any evidence of malignancy elsewhere in the ear.

Post-operative staging $\mathrm{CT}$ and magnetic resonance imaging scans showed no evidence of spread of disease. The patient underwent a revision modified radical mastoidectomy. The skin of the external auditory canal, posterior canal wall, malleus and incus was removed en bloc. Histological analysis demonstrated no evidence of residual tumour.

\section{Conclusion and lessons learned}

Squamous cell carcinoma of the tympanic membrane is a rare condition. The literature contains two previous reports but little guidance on appropriate management. Surgical resection offers optimal outcome if disease is limited to the tympanic membrane.

Spread of this tumour has not previously been reported. This may be due to the fact that the vascular and lymphatic drainage of the tympanic membrane is limited.

\section{A young woman with slowly progressive facial asymmetry}

J Thaj, A Bhattacharyya

From Whipps Cross University Hospital, London

\section{Introduction}

The diagnosis of vascular malformations of the head and neck is based on clinical behaviour and radiological features. In this report, we discuss a case of a vascular malformation in the buccal space, and its management.

\section{Case report}

A 23-year-old woman presented with a 12-year history of slowly progressive facial asymmetry. On examination, she had a diffuse swelling of the right cheek which was more prominent on smiling. Intra-orally, an irregular mass was palpable anterior to the retromolar triangle.

\section{Radiology}

Ultrasonography with Doppler showed a low-flow mixed echogenic lesion in the right cheek. A computed tomography scan revealed a heterogeneously enhancing mass in the right buccal space with indistinct margins, which was seen to infiltrate the masseter muscle.

\section{Management}

Although percutaneous image-guided sclerotherapy is currently the mainstay of treatment for venous malformations, there are cosmetic implications. Our patient opted for conservative management.

\section{Conclusion}

Management of venous vascular malformations can be challenging, especially in cosmetically sensitive areas.

\section{Paediatric session}

Chairman: Miss Hanna Burns

\section{Delayed airway obstruction in a child with a cutaneous} haemangioma

P Silva, J Goswamy, I A Bruce

From The Royal Manchester Children's Hospital

\section{Background}

The association between cutaneous haemangioma and subglottic haemangioma in children is well recognised. The frequency of concomitant cutaneous lesions varies between 30 and 50 per cent. Multiple therapeutic options have been proposed; however, there is increasing evidence to support the use of propranolol. We present a case of delayed airway obstruction in a child with multiple haemangiomas.

\section{Case report}

A four-month-old boy presented with multiple cutaneous haemangiomas in a beard-like distribution, without airway symptoms. Following two weeks of in-patient treatment with propranolol, he was discharged home on a maintenance dose, having demonstrated a modest reduction in the size of the cutaneous haemangiomas.

Four weeks later, he re-presented with biphasic stridor and significant respiratory distress, and underwent an emergency rigid airway endoscopy. A circumferential subglottic haemangioma was identified, and a tracheostomy was performed to safeguard the airway.

\section{Radiological findings}

On initial presentation, magnetic resonance imaging identified enlarged parotid and submandibular glands with high T2 fat-suppressed signals, features consistent with a congenital vascular malformation.

\section{Histological findings}

Intra-operative neck biopsies revealed features consistent with an infantile haemangioma.

\section{Conclusion}

This case highlights the potential for delayed-onset airway obstruction in the presence of multiple cutaneous haemangiomas.

The excellent response of infantile haemangiomas to propranolol is well documented, and this drug is now recommended as first-line treatment. Our case provides a cautionary note to the clinician that patients with multiple haemangiomas, such as those seen in PHACE syndrome (i.e. posterior fossa malformations, haemangiomas, arterial anomalies, coarctation of the aorta and other cardiac defects, and eye abnormalities), may present with delayed airway obstruction and may also be refractory to propranolol. 


\section{An unusual nasal mass}

R A Tasca, R Sharma, A Swift

From Alder Hey Children's NHS Foundation Trust, Liverpool

\section{Introduction}

Differentiated thyroid cancer metastasis to the orbit is an unusual presentation in the paediatric population.

\section{Case report}

A seven-year-old girl presented to the ENT department with a three-month history of left periorbital swelling and proptosis, without visual disturbance or associated pain.

The patient had undergone a right hemithyroidectomy at the age of three years for a thyroid swelling. Histological examination had been reported as showing a benign goitre.

An endoscopic transnasal biopsy of the nasal mass was performed under general anaesthetic. Biochemical investigation confirmed a euthyroid state, with no thyroid autoantibodies.

\section{Radiological findings}

Initial computed tomography and magnetic resonance imaging (MRI) scans of the head and orbits showed a soft tissue mass within the left nasal passage, involving the left ethmoid cells and breaching the medial wall of the orbit.

Subsequent MRI scans of the orbit and paranasal sinuses performed six and 12 months after ${ }^{131}$ I radioiodine treatment showed resolution of the solid component of the left frontoethmoidal mass.

\section{Histological findings}

Histological examination showed metastatic follicular thyroid carcinoma.

\section{Management}

Patient management consisted of a completion total thyroidectomy and two doses of post-operative ${ }^{131}$ I radioiodine treatment at 15-month intervals. At the time of writing, the patient was receiving thyroid replacement and alphacalcidol.

At the time of writing, it was planned to assess the response to the second ${ }^{131}$ I dose with an MRI scan six months later. If any residual frontoethmoidal disease was present, treatment would include surgical resection via an endoscopic or external approach.

Conclusion and lessons learned

Surgical resection should be deferred until the response to radioiodine treatment is assessed.

\section{An expanding neck mass in an infant with thrombocytopenia}

J Goswamy, I A Bruce, M P Rothera

From The Royal Manchester Children's Hospital

\section{Introduction}

We present a rare cause of a neck mass in an infant, highlighting the importance of early tertiary referral in all childhood cases of expanding neck mass.

\section{Case report}

A four-month-old boy presented with spontaneous periorbital bruising and a right-sided neck mass. Thrombocytopenia and anaemia required correction, and tracheostomy was undertaken to secure the airway.
Radiological findings

A computed tomography scan demonstrated a soft tissue mass in the right posterior triangle, extending to the skull base. A magnetic resonance imaging scan confirmed additional extension into the parapharyngeal space and nasopharynx.

\section{Histological findings}

Histological examination of a biopsy of the mass confirmed the presence of proliferating haemangioendothelioma.

\section{Management}

The histology result and the infant's ongoing consumptive coagulopathy led to the diagnosis of Kasabach-Merritt syndrome. Systemic steroids, vincristine and embolisation stabilised the platelet count and decreased tumour size, allowing extubation five days after embolisation.

\section{Conclusion and lessons learned}

Kasabach-Merritt syndrome is a rare childhood disorder characterised by purpura and thrombocytopenia associated with a vascular tumour. Life-threatening haemorrhage may result secondary to a consumptive coagulopathy caused by platelet trapping by the tumour. Kasabach-Merritt syndrome poses a diagnostic and therapeutic challenge, as the vascular tumour differs from the classical 'involuting' haemangioma of infancy. Response is variable. However, the tumour will involute if platelet consumption is arrested. Any case of an infant with a consumptive coagulopathy associated with thrombocytopenia should alert the clinician to the need to exclude Kasabach-Merritt syndrome.

\section{Obstructive sleep apnoea due to an expanding neck mass}

H Beer, S E Coupland, A J Donne

From Alder Hey Children's Hospital, Liverpool

\section{Introduction}

Epstein-Barr virus (EBV) infection is common, and presents with a spectrum of clinical manifestations and complications. We report a rare case of parapharyngeal and retropharyngeal abscess due to EBV, in which the histological appearance was difficult to interpret.

\section{Case report}

A two-year-old girl was referred with a four-week history of severe obstructive sleep apnoea, weight loss and left neck fullness. Despite medical therapy, significant oxygen desaturation episodes occurred, necessitating admission to the intensive therapy unit. A parapharyngeal abscess was identified on ultrasound and $20 \mathrm{ml}$ of pus aspirated. Two days later, a further collection was aspirated under ultrasound guidance and a drain left in situ. The neck swelling and symptoms persisted. Subsequent computed tomography scanning indicated a large, thin-walled, parapharyngeal and retropharyngeal abscess, so formal incision and drainage were performed. During this procedure, three large, level II lymph nodes were excised. The abscess did not yield pus, and an incisional biopsy was taken. Concurrent trans-oral aspiration was also negative. Post-operatively, magnetic resonance imaging demonstrated a thick-walled, deflated abscess cavity.

On haematoxylin and eosin staining, Reed-Sternberg cells were identified, suggesting lymphoma; on further analysis, DNA in situ hybridisation identified EBV. Further immunohistochemical staining allowed differentiation between lymphoma and infection. Retrospectively, EBV blood serology was found to be positive for immunoglobulins $\mathrm{M}$ and $\mathrm{G}$. 
Conclusion and lessons learned

Epstein-Barr virus infection can be difficult to differentiate from lymphoma unless the pathologist is a specialist in this field. Correct diagnosis is essential to prevent inappropriate chemotherapy. In the presented case, DNA in situ hybridisation and immunohistochemical staining for B-cell specific activator protein (PAX-5), cluster of differentiation 15 glycoprotein and epithelial membrane antigen (EMA) enabled accurate diagnosis.

\section{Obstructive supraglottic soft tissue mass: case report \\ P D Hettiarachchi, S C Leong, R W Clarke \\ From Alder Hey Children's Hospital, Liverpool}

\section{Case report}

A three-year-old boy first presented at the age of five weeks with a harsh inspiratory stridor associated with a severe tracheal tug and sternal recession.

He underwent bronchoscopy, during which a large, smooth, supraglottic mass was discovered obstructing the trachea. An emergency tracheostomy was required to protect the airway.

Magnetic resonance imaging (MRI) and computed tomography scanning demonstrated a fibrotic mass at the level of the hyoid bone, with no cystic or vascular component and no bony abnormalities or lymphadenopathy.

Histopathological examination of successive biopsies found chronic inflammatory and granulation tissue with patchy haemorrhage and mild fibrosis, with some samples showing increased numbers of spindle fibres. There was no evidence of malignancy. A diagnosis of infantile myofibroma was made. Due to the location of the lesion, excision was held as a last resort. The patient underwent laser debulking and has since been decannulated; at the time of writing he was stable, and undergoing long-term follow up with periodic check endoscopies.

\section{Discussion}

Dr Siddiqui noted that the lesion's MRI signal could indicate many tissues, including blood, dense fibre and malignancy. Dr Sandison agreed that infantile myofibroma was the most appropriate diagnosis based on the histology. These benign lesions, while being the commonest fibrous tumours of infancy, are sufficiently rare to be initially misdiagnosed. Thirty per cent involve the head and neck, and the literature suggests that surgical treatment is widely accepted. However, as many such lesions have also been shown to spontaneously regress, conservative management remains a viable option, although emergency interventions may be required to avoid acute complications.

\section{Rhinology session}

Chairman: Mr David Roberts

\section{An unusual source of recurrent epistaxis after parotidectomy and mastoidectomy}

H A Elhassan, N Gibbins, S Watts

From the Royal Sussex County Hospital, Brighton

\section{Case report}

A 64-year-old retired solicitor presented to the accident and emergency department on five occasions over the course of two months with brief, profuse bouts of epistaxis from the left side of the nose. He was treated with silver nitrate cauterisation, overnight observation and left sphenopalatine artery ligation. He re-presented two weeks later with bleeding from both the nose and the left ear, through a tympanic membrane perforation.

The patient's medical history included type I diabetes, left mastoidectomy for malignant otitis externa one year previously, and left parotidectomy with radiotherapy for a 'non-malignant' lump 30 years ago.

Examination revealed blood in the left ear canal and nostril, and at the left torus tubarius of the nasopharynx. Neurological examination was normal.

\section{Radiology}

A magnetic resonance imaging scan revealed a mass just behind the left fossa of Rosenmuller, involving the internal carotid artery. Carotid angiography confirmed a $30 \mathrm{~mm}$ diameter, subcranial aneurysm of the left internal carotid artery just inferior to the petrous temporal bone.

\section{Management}

The patient underwent embolisation of the left internal carotid artery under sedation. Recovery was unremarkable, with no neurological deficit or further bleeding.

\section{Conclusion}

Internal carotid aneurysms are rare and of uncertain aetiology. Middle-ear infection, irradiation and mastoid surgery have been implicated in the development of aneurysms of the petrous segment. Presentation depends on the direction of aneurysm expansion; signs and symptoms may include epistaxis, otorrhagia, cranial nerve palsies, Horner's syndrome and pulsatile tinnitus.

Magnetic resonance imaging and magnetic resonance angiography may be diagnostic, but carotid angiography is the 'gold standard'. Successful treatment of internal carotid aneurysms involves endovascular occlusion, which has superseded surgical ligation.

\section{A persistently enlarging maxillary lesion}

L Ferguson, C R Butler, G Alusi

From Barts and The London Hospital NHS Trust

\section{Introduction}

We report the case of a 36-year-old man presenting with a left maxillary lesion which proved to be a histological diagnostic dilemma.

\section{Case report}

This man presented with a one-year history of a painful, leftsided, maxillary swelling intermittently treated with antibiotics in the community. Clinically, there was an obvious swelling displacing the left alar of the nose. Nasendoscopic examination was normal.

\section{Radiology}

Magnetic resonance imaging showed a naso-alveolar lesion with extensive, ill-defined signal changes in the adjacent soft tissues which extended onto the alveolar gingiva and supero-laterally into the fat plane adjacent to the naso-labial facial muscles. Bone remodelling was noted in the midline at the anterior nasal spine. The infra-orbital nerve was normal.

\section{Management}

An initial biopsy revealed chronic inflammatory changes, with no dysplasia or malignancy. Immunology investigations 
were normal. A 'watch and wait' policy was adopted. However, increasing lesion size over an eight-month period prompted a further excisional biopsy, which was followed by corticosteroid treatment.

\section{Histology}

Histological examination demonstrated a tumefactive fibroinflammatory lesion considered part of a broader fibro-sclerosing syndrome akin to retroperitoneal fibrosis. At the time of writing, disease differentiation remained a challenge; other possible diagnoses included inflammatory lesions of infective aetiology, nodular fibromatoses, inflammatory myofibroblastic tumours and pseudo-sarcomatous lesions.

\section{Conclusion}

Tumefactive fibro-inflammatory lesions are extremely rare tumours of the head and neck region, of unknown aetiology. The mainstays of treatment include immune modulatory medication.

\section{Beware the nasal polyp!}

J Howard, A Taghi, P Clarke

From Charing Cross Hospital, London

\section{Introduction}

Inverted papillomas are relatively uncommon tumours which constitute $0.5-4$ per cent of all nasal neoplasms. They have a polypoid appearance, a male preponderance and a predilection for the lateral nasal wall. A tendency to recurrence and an association with malignancy are both well recognised.

\section{Case report}

A 28-year-old man presented with a firm swelling of the nasolacrimal sac, and was found to have a fleshy, polypoid mass filling the anterior left nostril. Biopsies were reported as consistent with inverted papilloma, and he was managed with a lateral rhinotomy and local resection.

Recurrences on the posterior septum, one and two years later, were also resected. At this point, the patient was referred to a tertiary centre for review. Imaging showed thickening of the lateral nasal wall. A further biopsy showed atypical features, prompting review of the previous biopsies. Upon review, the initial biopsies were seen to show squamous cell carcinoma (SCC). Resection was undertaken via a midfacial degloving procedure, and the patient received post-operative radiotherapy.

\section{Conclusion and lessons learned}

This case highlights some of the hazards of diagnosing a rare tumour with uncertain biology. Lesions that are atypical clinically are probably best managed by a multi-disciplinary team at tertiary level. Mr Roberts commented that if a polyp invades the nasolacrimal system it is an SCC and not an inverted papilloma. Professor Michaels noted that in most cases of inverted papilloma it is difficult to confidently rule out SCC; thus, clinicians should have a high index of suspicion. It is much rarer to have an inverted papilloma transform into an SCC

Severe, recurrent epistaxis caused by nasal septal angiofibroma: role of embolisation and treatment

\author{
A Riskalla, R Kuchai, C East \\ From the Royal National Throat, Nose and Ear Hospital, London
}

Case report

A 28-year-old man was initially referred with severe, uncontrollable epistaxis. Eight years previously, he had had a nasal septal haemangioma excised. Prior to the current presentation, he had been admitted to three other tertiary hospitals for epistaxis, had been packed several times, and had undergone sphenopalatine artery ligation and adenoidectomy.

Clinically, there was a purple mass in the right anterior aspect of the nasal septum. Magnetic resonance imaging confirmed recurrence of the haemangioma. Right anterior and posterior ethmoid artery ligation was unsuccessful. Radiological embolisation of the right facial and superior alveolar artery reduced the epistaxis to an intermittent ooze.

Under general anaesthesia, the septal mass was excised through an external rhinoplasty approach. A conchal cartilage graft was used to reconstruct the septal defect.

\section{Discussion}

The mass, which was initially thought to be a nasal septal haemangioma, was found by Professor Michaels to have the characteristics of an angiofibroma. Professor Michaels stated that such a lesion was very rare, and thus unexpected, on the nasal septum. Mr Roberts added that the nasal septum is also an extremely rare site for an angiofibroma, and that embolisation followed by surgical excision was the treatment of choice. There have been seven previously reported cases of an angiofibroma located on the nasal septum.

\section{A rare sarcoma presenting as sinusitis}

A Taghi, M Bentley, H Saleh

From Charing Cross Hospital, London

\section{Introduction}

Myxofibrosarcoma of the nasal cavity and paranasal sinuses is an extremely rare and aggressive tumour with a high propensity for local recurrence and distant metastasis. This tumour was first described in 1964 and there have been few cases reported in the world literature. Histologically, it is a myxoid variant of malignant fibrous histiocytoma. Treatment is by surgery and radiotherapy, but the prognosis remains poor.

\section{Case report}

A 51-year-old woman presented with a history of pain around her left eye and left maxillary sinus, which rapidly progressed to diplopia and mild proptosis. The patient denied any nasal symptoms, and nasal examination was unremarkable. Biopsies showed atypical spindle cells which were diagnostic of a low grade myxofibrosarcoma.

Imaging showed a left maxillary mass which had expanded the bony antrum and displaced the orbit superiorly. There was no evidence of aggressive bony erosion.

The patient underwent an extended maxillectomy via a midfacial degloving approach, with preservation of the orbit. This was followed by adjuvant radiotherapy.

Subsequent Semon Club discussion emphasised the histological variance of tumours within the nasal cavity, and noted that the insidious growth of tumour within the paranasal sinus may lead to difficulties in early diagnosis. 